\title{
La dosimétrie passive en France en 2010
}

\author{
A. BIAU
}

Pour faire l'état de la situation actuelle, la source d'informations la plus complète est le Rapport IRSN/DRPH/2010-4 intitulé « La Radioprotection des travailleurs Bilan 2009 de la surveillance des travailleurs exposés aux rayonnements ionisants ». (Ce rapport est consultable sur le site "www.irsn.fr") (IRSN, 2010). Il correspond, en plus détaillé, au bilan annuel transmis par l'OPRI au ministère du Travail évoqué au chapitre 13.

Si l'on compare la situation de 2010 à celle de 2000, on constate les principales évolutions suivantes.

\subsection{Les laboratoires de dosimétrie passive}

Les laboratoires d'EDF et Philips ont disparu sous leur forme de 2000 pour s'intégrer au LCIE devenu LCIE Landauer puis Landauer Europe. L'IPSN et l'OPRI ayant fusionné en 2002, les deux laboratoires se sont regroupés en un seul laboratoire de l'IRSN. Ce laboratoire a une structure de «business unit » dirigée par Simon Tourard à l'intérieur du Service de dosimétrie externe (SDE) au sein de la Direction de radioprotection de l'homme (DRPH).

Parallèlement un nouveau laboratoire privé, Dosilab, est apparu.

Les autres laboratoires, AREVA La Hague et Marcoule, CNRS IN2P3 Orsay et Strasbourg et le SPRA ont gardé leurs structures mais ont changé de technique de dosimétrie.

En effet l'autre changement majeur est l'abandon de la dosimétrie photographique obligatoire au profit d'autres techniques toutes basées sur la luminescence de matériaux après irradiation, TLD, OSL et RPL.

Le tableau I fait la synthèse des laboratoires de dosimétrie passive et des techniques utilisées. 
TABLEAU I

Les différents laboratoires de dosimétrie passive en France en 2009.

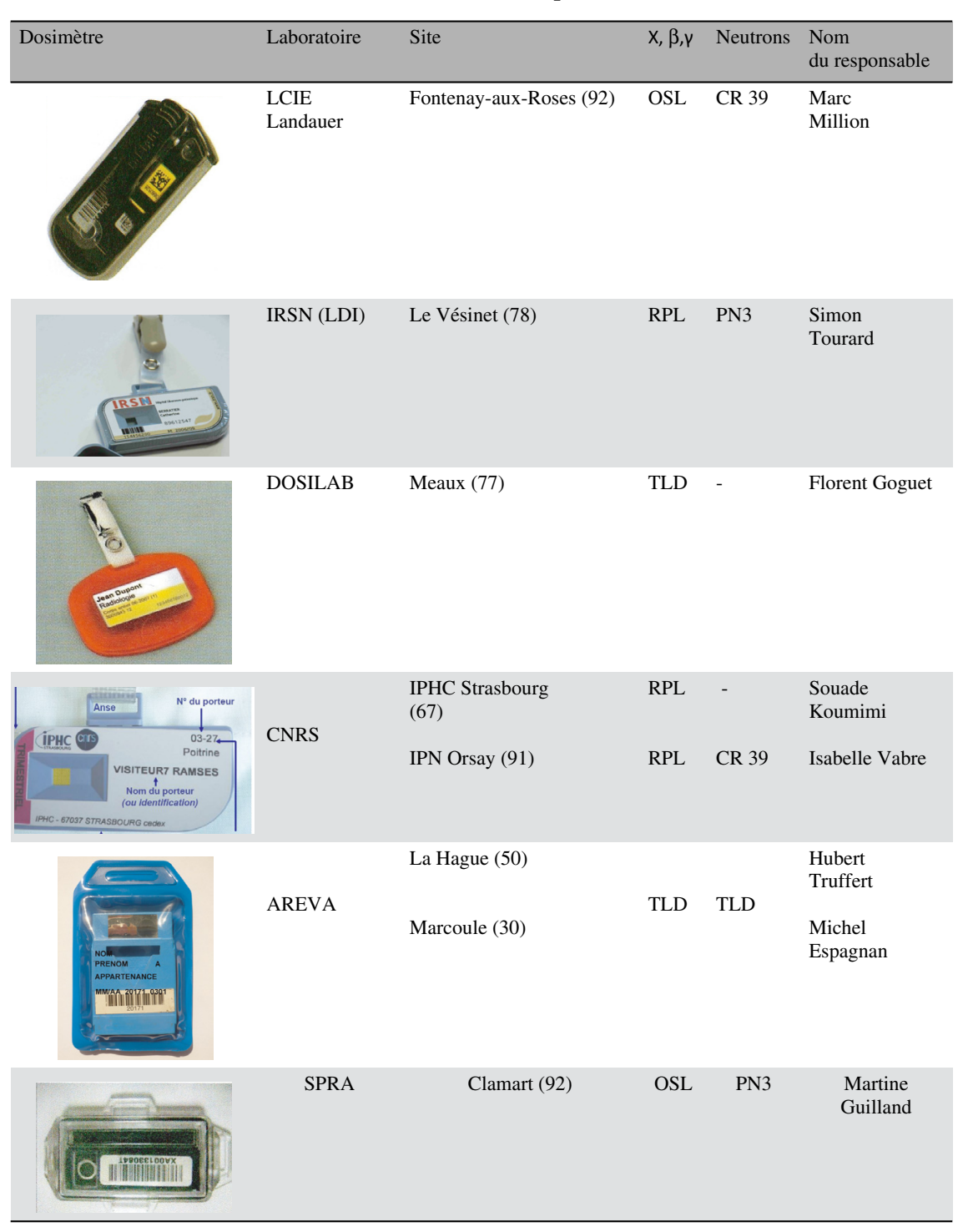




\section{2. Évolution de SISERI}

En 2000, SISERI était encore en état de projet, à partir de la création de l'IRSN en 2002, sa mise en œuvre s'est accélérée pour atteindre un régime de croisière en 2009. Par ailleurs, avec l'amélioration de la transmission des résultats de dosimétrie passive par les laboratoires, SISERI dispose d'une énorme quantité de résultats dosimétriques individuels constituant une remarquable base de données. Pour optimiser l'exploitation de ces données, SISERI a créé une nouvelle codification des activités, des métiers et des sources de rayonnements qui devrait permettre, à partir de l'exploitation des résultats de l'année 2000 (en 2001) d'établir des statistiques beaucoup plus précises et détaillées que celles établies à partir de la codification de 1975 en vigueur jusqu'en 2009. Des efforts conséquents ont été également réalisés en termes d'information sur la réglementation et le fonctionnement au jour le jour du système SISERI. La figure 15.1 illustre clairement le fonctionnement de SISERI.

Tout ce qui concerne le fonctionnement et les résultats de SISERI peut être consulté sur les sites de l'IRSN ou celui, spécifique de SISERI, www.irsn.fr/siseri.

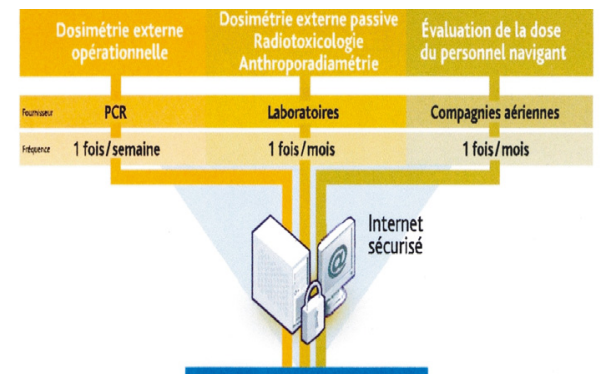

\section{BASE DE DONNEEES SISERI}

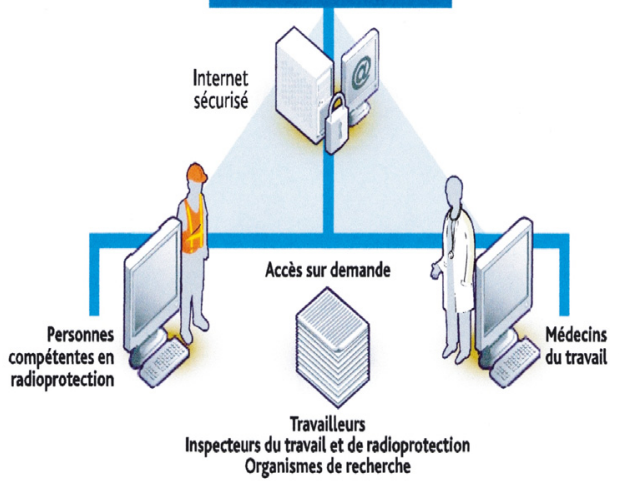

Figure 15.1 - Schéma de fonctionnement de SISERI (IRSN, 2010). 


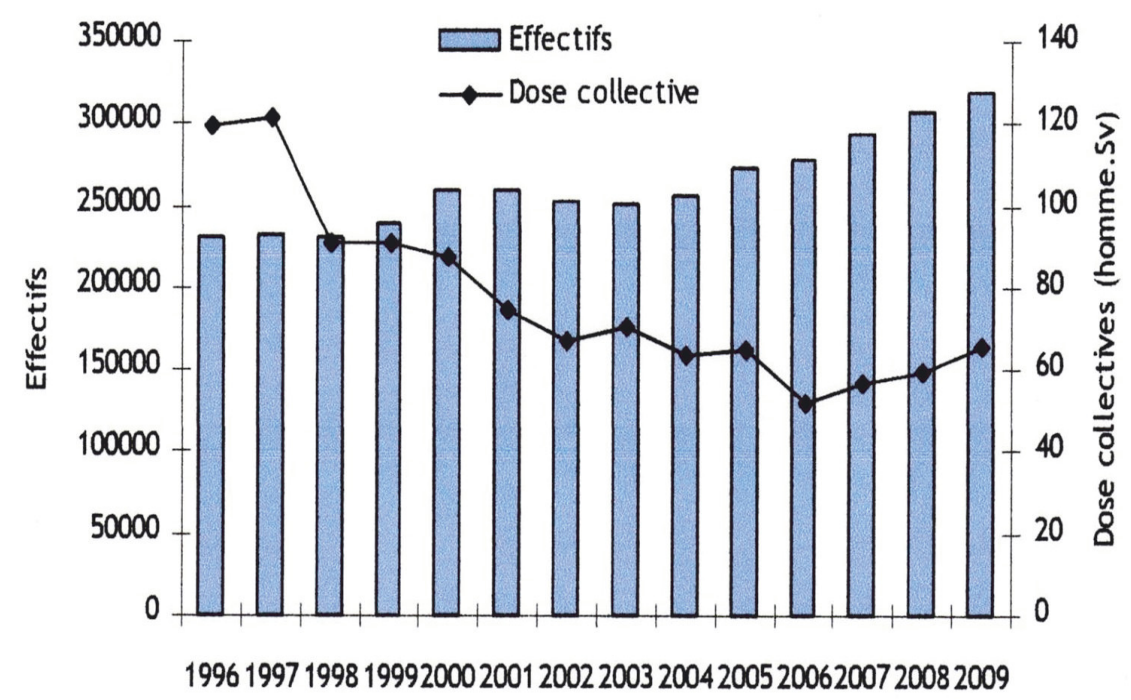

Figure 15.2 - Évolution du nombre de personnes surveillées et de la dose collective (somme des doses individuelles) entre 1996 et 2009 (IRSN, 2010).

\subsection{Bilan dosimétrique 2009}

Les bilans dosimétriques de 2009 portent sur l'ensemble des travailleurs exposés aux rayonnements ionisants en France et suivis par les laboratoires de dosimétrie passive évoqués ci-dessus. Ces bilans qui sont transmis réglementairement, comme le faisait le SCPRI puis l'OPRI, présentent des résultats plus détaillés en raison notamment de l'évolution de SISERI, et sont publiés dans le rapport IRSN cité ci-dessus (figure 15.2).

Le nombre de personnes surveillées est resté relativement stable de 2000 à 2004 puis a augmenté légèrement et régulièrement pour atteindre 320000 personnes en 2009. Cette augmentation peut s'expliquer par la réorganisation des conditions de travail à la suite de l'application du décret de 2003 entrainant la mise en place de la dosimétrie opérationnelle et par la même occasion de la dosimétrie passive.

La dose collective qui a diminué nettement entre 1996 et 2004 en raison, notamment, de la mise en place de la dosimétrie opérationnelle, est restée relativement stable entre 2004 et 2009 avec une très légère augmentation de 2007 à 2009 sans doute liée à l'augmentation des effectifs. 
Avant 1996, il n'y avait pas de statistique globale car SISERI n'était pas opérationnel. Des bilans dosimétriques étaient effectués par laboratoire car il n'était pas possible d'additionner systématiquement ces données. En effet, une même personne pouvait, au cours d'une année, être suivie en dosimétrie par des laboratoires différents selon les entreprises où elle travaillait.

Le tableau II et la figure 15.3 ci-dessous montrent l'évolution des doses supérieures aux limites de doses de 20 et 50 mSv de 1996 à 2009.

\section{TABLEAU II}

L'évolution des doses supérieures aux limites de doses de 20 et 50 mSv de 1996 à 2009.

\begin{tabular}{|ccc}
\hline Année & Nombre de doses supérieures à $20 \mathrm{mSv}$ & Nombre de doses supérieures à $50 \mathrm{mSv}$ \\
\hline 1996 & 905 & 63 \\
\hline 1997 & 340 & 43 \\
\hline 1998 & 322 & 42 \\
\hline 1999 & 177 & 26 \\
\hline 2000 & 69 & 27 \\
\hline 2001 & 112 & 22 \\
\hline 2002 & 79 & 14 \\
\hline 2003 & 100 & 18 \\
\hline 2004 & 51 & 11 \\
2005 & 40 & 7 \\
2006 & 26 & 5 \\
\hline 2007 & 22 & 2 \\
\hline 2008 & 16 & 4 \\
\hline 2009 & 14 & 2 \\
\hline
\end{tabular}

Comme rappelé dans le chapitre 4, la limite annuelle de dose n'a été fixée à $20 \mathrm{mSv}$ qu'en 2003 mais cette limite a été prescrite dans la Directive européenne 96/29 de 1996, c'est la raison pour laquelle, par anticipation, le nombre de doses supérieures à $20 \mathrm{mSv}$ a baissé dès 1997 (340 contre 905 en 1996) et de façon régulière jusqu'en 2003 en raison, notamment, de la mise en œuvre de la dosimétrie opérationnelle de façon mieux organisée dans le domaine nucléaire. À partir de 2004, la parution du décret de mars 2003 officialisant le passage à la limite de $20 \mathrm{mSv}$, entraîne la baisse accrue du nombre de ces dépassements (51 en 2004 contre 100 en 2003) qui se prolonge jusqu'en 2009 (14 cas). Une même évolution pour les doses supérieures à $50 \mathrm{mSv}$ est observée avec une baisse régulière jusqu'en 2003 et encore plus nette à partir de 2004 pour ne compter que 2 cas en 2009. 
Enfin l'examen détaillé du rapport IRSN cité ci-dessus montre que l'évolution des doses collectives et des doses supérieures à $20 \mathrm{mSv}$ dans les quatre principaux domaines d'activité confirme que c'est bien dans le domaine nucléaire et à un degré moindre la radiographie industrielle, souvent pratiquée en milieu nucléaire, que les diminutions de dépassements de dose sont les plus nettes.

Dans le milieu médical et vétérinaire, la chute a été plus progressive mais significative malgré l'augmentation des effectifs qui explique une légère augmentation de la dose collective dans ce domaine.

\subsection{Réglementation, évolution dans les prochaines années}

L'amélioration de la situation dosimétrique s'est accentuée nettement ces vingt dernières années mais en pratique l'amélioration n'a jamais cessé depuis les années 1970. Depuis la fin des années 1990, les réformes de la réglementation, les progrès techniques en dosimétrie passive et opérationnelle et la réorganisation des instances de radioprotection sont à l'origine de cette accentuation de l'amélioration (paragraphe ci-dessus). Il est probable que les limites de doses restent au niveau actuel pour quelques années, par contre il est vraisemblable que des améliorations seront apportées à certains aspects pratiques comme la réglementation du zonage qui doit être plus lisible avec des conséquences sur l'utilisation des dosimètres. Ces aménagements seront facilités par le rôle de plus en plus déterminant des Personnes compétentes en radioprotection dont la formation et les obligations réglementaires seront clarifiées.

\section{RÉFÉRENCE}

IRSN (2010) Institut de radioprotection et de sûreté nucléaire, Rapport DRPH/DIR/2010-4. 